\title{
Albinoni für Laute. Transkriptionen italienischer Musik für die Münchner Prinzessinnen Josepha Maria und Maria Josepha von Bayern
}

\section{Albinoni for Lute. Transcriptions of Italian Music for the Munich Princesses Josepha Maria and Maria Josepha of Bavaria}

Berthold Over / over@uni-mainz.de

Department of Musicology, Johannes Gutenberg University Mainz, DE

\begin{abstract}
Between 1751 and 1764 a large quantity of lute music was copied for the Bavarian princesses Josepha Maria and Maria Josepha. All the manuscripts seem to be lost, but relatively detailed copyist bills give us an impression of the repertoire. In general, the repertoire did not consist of original works, but of arrangements reflecting the specific requirements of the electoral players and showing the arrangement practices of the time. It is remarkable that only a few works by prominent composers of the Munich Hofkapelle were in this repertoire. More are found from genuine lute and other composers. If we have a look at Italian composers, there were several compositions in the repertoire. Amongst them are six concerts by Albinoni in four parts, but a similar group of works is not found amongst Albinoni's musical output. Thoughts on the identity of Albinoni's concerts, on the Munich repertoire and on the transfer of Italian music conclude this insight in a lost corpus of lute music.
\end{abstract}

\section{Keywords}

Tomaso Albinoni, lute music, Munich court, Josepha Maria of Bavaria, Maria Josepha of Bavaria 
Die Blüte der Lautenmusik verortet man gemeinhin im 16. Jahrhundert, allenfalls Silvius Leopold Weiß ist sozusagen als einer der letzten Vertreter von Lautenvirtuosen bekannt. Dabei war die Laute als Instrument des bürgerlichen und vor allem adligen Musizierens bis weit in die zweite Hälfte des 18 . Jahrhunderts verbreitet. ${ }^{1}$ Als moralisch legitime Beschäftigung, das otium, die Mußestunden zu füllen, war die Musik seit jeher aus dem an frei verfügbarer Zeit reichen adligen Alltag nicht wegzudenken. Saiteninstrumenten wurde dabei als quasi „apollinischen“ Instrumenten im Gegensatz zu den „dionysischen“ Blasinstrumenten seit Castiglione der Vorzug gegeben. Dies lässt sich auch am Münchner Hof beobachten. Während Max Emanuel von Bayern Viola da gamba spielte, erlernten sein Erstgeborener, Kurprinz Karl Albrecht, sowie seine Brüder Ferdinand Maria und Clemens August die Laute. ${ }^{2}$ Karl Albrechts Kinder pflegten wiederum die Instrumente der Vorfahren: Max III. Joseph spielte Viola da gamba, ${ }^{3}$ seine Schwestern Maria Josepha (1734-1776) und Josepha Maria (1739-1767) wurden in Laute unterrichtet - um hier nur einige wenige Beispiele zu nennen.

Es ist relativ wenig über das rezipierte Repertoire bekannt. In Bezug auf Karl Albrecht machte mich Frank Legl auf ein Dokument aufmerksam, das er mir freundlicherweise zur Verfügung stellte, aus dem wir erfahren, dass der Lautenist Johann Joachim Setzkorn, ein Kammerdiener der Kurfürstin Maria Amalia, Opern für Laute transkribierte - und hier kommt Albinonis Name zum ersten Mal ins Spiel: Er habe nämlich für den 1745 verstorbenen Karl Albrecht neben Trios und Konzerten ungenannter Komponis-

1 Ein Beispiel eines Laute spielenden Adligen, der sogar einige Berühmtheit erlangt hat, ist Johann Adam Graf von Questenberg. Vgl. PERTUTKOVÁ, Jana. Der glorreiche Nahmen Adami. Johann Adam Graf von Questenberg (1678-1752) als Förderer der italienischen Oper in Mähren. Wien: Hollitzer, 2015, S. 143-147. Zur Laute im 18. Jahrhundert vgl. FARSTAD, Per Kjetil. German Galant Lute Music in the 18th Century. A Study of the Period, the Style, Central Lutenists, Ornaments, Idiomatic, and Problems that Arise When Adapting Lute Music from this Period to the Modern Eight-Stringed Classical Guitar. Göteborg: Göteborg Univ., Dep. of Musicology, 2000. Ders. German Galant Lute Music. Guitar Review, 2001, 123, S. 10-23. LEGL, Frank. „der alte Weiß spielte“ - Laute und Theorbe in der zweiten Hälfte des 18. und zu Beginn des 19. Jahrhunderts. In Laute und Theorbe. Symposium im Rahmen der 31. Tage Alter Musik in Herne 2006. Stadt Herne (Hrsg.), Konzeption und Redaktion Christian Ahrens - Gregor Klinke. München und Salzburg: Katzbichler, 2009, S. 30-49. PÄFFGEN, Peter. Ein Artliches Lob der Lauten. Blüte und Niedergang von Laute und Lautenspiel im 16.-18. Jahrhundert. Concerto. Das Magazin für Alte Musik, 1985, 2, S. 48-55.

2 WERR, Sebastian. Politik mit sinnlichen Mitteln. Oper und Fest am Münchner Hof (1680-1745). Köln u.a.: Böhlau, 2010, S. 56-59. OVER, Berthold. ,...sotto l'Ombra della Regina di Pennati‘. Antonio Vivaldi, Kurfürstin Therese Kunigunde von Bayern und andere Wittelsbacher. In Italian Opera in Central Europe 1614-1780. Bd. 3: Opera Subjects and European Relationships. Norbert Dubowy - Corinna Herr - Alina Zorawska-Witkowska (Hrsg.). Berlin: BWV - Berliner Wissenschafts-Verlag, 2007, S. 251-297: 259. MÜNSTER, Robert. Die Musk am Hofe Max Emanuels. In Kurfürst Max Emanuel. Bayern und Europa um 1700. Bd. 1: Zur Geschichte und Kunstgeschichte der Max-Emanuel-Zeit. Hubert Glaser (Hrsg.). München: Hirmer, 1976, S. 295-316: 305f. ZEDLER, Andrea. Alle Glückseligkeit seiner Education dem allermildesten Ertz-Hause Oesterreich zu dancken“. Hofstaat, Bildung und musikalische Unterweisung des bayerischen Kuprinzen Karl Albrecht in Graz (1712-1715). Historisches Jahrbuch der Stadt Graz, 2012, 42, S. 337-366: 356. Herzog Ferdinand Maria von Bayern spielte auch Flöte. WERR, op. cit., S. 56. MÜNSTER, Robert. Herzog Clemens Franz von Paula von Bayern (1722-1790) und seine Münchner Hofmusik. Tutzing: Schneider, 2008, S. 9.

3 SADGORSKI, Daniela. Andrea Bernasconi und die Oper am Münchner Kurfürstenhof 1735-1772. München: Herbert Utz Verlag, 2010, S. 31-32. 
ten Opern von Albinoni und Torri für Laute arrangiert. Der Passus in der Supplik vom 6. August 1760 lautet: $^{4}$

„[...] weilen Sr: Majestätt Kayser Carl den Siebenten Allerglorwürdigsten angedenckens ich in die 20. ganzer Jahr lang in der Lauten allerunterth[äni]st zu bedienen die Aller Höchste Gnad gehabt, wie auch von unterschiedlichen Maystern so wohl Trio iber etliche hundert, wie auch Concertten, als auch etliche Opera von Mr: Albinoni, als auch von Mr: Torri auf der Lauten habe Transponieren müssen."

Etwa 10 Jahre vor der Supplik, 1751, beginnt eine Reihe von Abrechnungen für Lautenmusik, die für Töchter Karl Albrechts, die Prinzessinnen Maria Josepha und Josepha Maria, kopiert wurde. ${ }^{5}$ Während 1751 die ersten Kopien für die damals 17-jährige Maria Josepha hergestellt wurden, kam 1754 die 15-jährige Josepha Maria hinzu. Diese Abrechnungen laufen bis zur Heirat der Prinzessinnen nach Baden (Maria Josepha, 1755) und Wien (Josepha Maria, 1765). Unter diesen Dokumenten finden sich auch solche, die zeigen, dass für die Prinzessinnen eine Reihe von Lauten erworben wurde, darunter ein Instrument von Wendelin Tieffenbrucker. ${ }^{6}$ In den Abrechnungen begegnet uns Albinoni zum zweiten Mal: Vom 5. August 1754 liegt eine Rechnung vor, in der es heißt:

\section{„2. Lauten Bücher [...] von Mr: Ferrandini et Mr: Albinoni Concertten“7}

Ein drittes Mal finden wir Albinoni in einer Rechnung vom 21. Mai 1755:

„Mehr von Monsr: Ferrandini .24. Concert, und von Mons: Albinoni .6. Concert, bestehen in .2. Violin, Viola, und Baß" ${ }^{\text {“8 }}$

Diese zuletzt genannten Konzerte stellen wahrscheinlich die Vorlagen für die Lautentranskriptionen dar, denn es finden sich in der Abrechnung ebenfalls Kompositionen anderer Komponisten, die mit Lautentranskriptionen des Vorjahrs in Verbindung gebracht werden können:

\footnotetext{
4 D-Mhsa, Kurbayern HR I, Fasz. 41, Nr 58/ ${ }^{\mathrm{Q}-\mathrm{z}}$. Zu Johann Joachim Setzkorn vgl. auch LEGL, Frank. Johann Joachim Setzkorn und ein Corpus verschollener Musik für und mit Laute. In Die Laute. Jahrbuch der Deutschen Lautengesellschaft (i.Vorb.). An dieser Stelle möchte ich Frank Legl sehr herzlich für seine zahlreichen Anregungen danken.

5 Die Abrechnungen befinden sich in D-Mhsa, Kurbayern Hofzahlamt (KBH) 1663 (1751), 1699 (1753), 1718 (1754), 1733 II (1755), 1764 (1757), 1781 (1758), 1800 (1759), 1818 (1760), 1833 (1761), 1850 (1762), 1870 (1763), 1883 (1764).

6 D-Mhsa, KBH 1781, Dok. 115 (1758).

7 D-Mhsa, KBH 1718, Dok. 133.

8 D-Mhsa, KBH 1733 II, Dok. 144.
} 
Albinoni für Laute. Transkriptionen italienischer Musik für die Münchner Prinzessinnen ...

\begin{tabular}{|c|c|c|c|}
\hline 5. August 1754 & & 21. Mai 1755 & \\
\hline "Camerloher" & "Second Lauten Buch" & "Camerloher" & $\begin{array}{l}\text { "23. Trio bestehen in } \\
\text { zwey Violin und Bass" }\end{array}$ \\
\hline $\begin{array}{l}\text { Ferrandini, Giovanni } \\
\text { Battista / Albinoni, } \\
\text { Tomaso }\end{array}$ & $\begin{array}{l}\text { "2. Lauten Bücher [...] } \\
\text { von Mr: Ferrandini et } \\
\text { Mr: Albinoni Concertten" }\end{array}$ & $\begin{array}{l}\text { Ferrandini, Giovanni } \\
\text { Battista / Albinoni, } \\
\text { Tomaso }\end{array}$ & $\begin{array}{l}\text { "Mehr von Mons: } \\
\text { Ferrandini .24. Concert, } \\
\text { und von Mons: Albinoni } \\
\text {.6. Concert, bestehen } \\
\text { in .2. Violin, Viola, und } \\
\text { Bass" }\end{array}$ \\
\hline Kropfgans, Johann & "6. Trio auf die Lauten" & Kropfgans, Johann & $\begin{array}{l}\text { "6. Trio mit Violin und } \\
\text { Bass" }\end{array}$ \\
\hline $\begin{array}{l}\text { Setzkorn, Ferdinand } \\
\text { Maria }\end{array}$ & "6. Lauten Parthien" & $?$ & $\begin{array}{l}\text { "ein Solo buch mit } 6 . \\
\text { Parthyen bestehen in } \\
\text { Violin und Bass" }\end{array}$ \\
\hline$?$ & $\begin{array}{l}\text { "ein Lauten Buch } \\
\text { dari[n]nen Solo von } \\
\text { unterschiedtlichen } \\
\text { Meystern" }\end{array}$ & $?$ & $\begin{array}{l}\text { "ein buch mit Violin und } \\
\text { Bass" } \\
\text { und/oder } \\
\text { "ein Solo buch mit Violin } \\
\text { et Bass" }\end{array}$ \\
\hline$?$ & $\begin{array}{l}\text { "2. Lauten Bücher } \\
\text { so wohl Trio als } \\
\text { auch Parthien, von } \\
\text { unterschiedlichen } \\
\text { Meystern" }\end{array}$ & $?$ & $\begin{array}{l}\text { "im buch ein buch mit } \\
\text { Trio besteht in zwey } \\
\text { Violin und Bass" }\end{array}$ \\
\hline
\end{tabular}

Beim Rechnungseintrag zu den Kompositionen des Lautenisten Kropfgans finden sich zusätzlich Hinweise auf die Bearbeitungspraktiken, die zeigen, dass seine Kompositionen ursprünglich die Triobesetzung Lt, Vl, B aufwiesen, und die zweite Laute wohl aus der Violinstimme gewonnen wurde:

„ist nur die Prim: Lauten von Mr: Kropffgans Componiert“

„Die Second habe auf die Lauten Transponiert und selbige 3 mahl, wie auch die Erste Lauten .2. mahl abschreiben lassen"

Dieser hier vorgestellte Repertoireausschnitt ist symptomatisch für das gesamte für München kopierte Repertoire: Es wurden Kompositionen mit Solo oder Triobesetzung für ein oder zwei Lauten arrangiert. Auch Konzerte wurden entsprechend angepasst. Die Komponisten können entweder ausgewiesene Spezialisten für Laute sein (Johann Kropfgans, Adam Falckenhagen, Charl Durant usw.) oder völlig unabhängig davon komponiert haben (Tomaso Albinoni, Giovanni Battista Ferrandini, Georg Christoph Wagenseil usw.). Das Repertoire wurde nur unwesentlich durch Münchner Hofkomponisten bestimmt. Neben den Lautenkonzerten Ferrandinis, deren Vorlagen verschollen sein dürften, da sich keine 24 wie auch immer besetzte Konzerte unter seinen Werken ausmachen lassen, ${ }^{9}$

9 Es sind hingegen Quartette in der Besetzung zwei Violinen, Viola und Bass überliefert. BÖHMER, Karl. 
findet sich nur ein weiteres Trio des Kammermusikdirektors; von Hofkapellmeister Andrea Bernasconi, der immerhin die Prinzessinnen in Musik unterrichtete, ${ }^{10}$ lediglich zwei Trios. Hingegen finden wir Kompositionen von weniger prominenten Mitgliedern der Hofkapelle, bzw. des Hofstaats: Der Erste Geiger des Münchner Hoforchesters, Wenzeslaus Wodiczka hatte 175824 Sinfonien für die Hofkapelle komponiert, ${ }^{11}$ von denen aller Wahrscheinlichkeit nach 1759 zwölf in bearbeiteter Form kopiert wurden. Wolf Jakob Lauffensteiner, der 1754 verstorbene Münchner Lautenist, ist mit drei Trios vertreten. Ferdinand Maria Setzkorn, maßgeblicher Bearbeiter und Sohn des Johann Joachim Setzkorn, legte mit 120 Stücken die größte Zahl an Kompositionen vor, die zu einem großen Teil nicht Originalkompositionen für Lauten waren. ${ }^{12}$ Schließlich waren Kompositionen von Kurfürst Max III. Joseph von Bayern im Repertoire (vier Quadro, vier Sonata à Quadro, zwölf Trios), die entsprechend bearbeitet wurden.

Im Regelfall wurden Trios, Quartette, Sinfonien, Konzerte so umgeschrieben, dass sie für zwei oder drei Lauten und ggf. ein kleines Ensemble spielbar wurden. Ferdinand Maria Setzkorns sechs Divertimenti für Flöte, zwei Violinen und Bc wurden für drei Lauten und Bc eingerichtet („sowohl die .6. Prim als die Second als auch die .6. Flauto auf die Lauten Transponiert. die .4. Sti $[$ m]men aus der Spart ingleichen die .3. Lauten Sti $[$ m] men habe Copieren lassen"). ${ }^{13}$ Dabei machte man vor genuin für Laute geschriebenem Repertoire nicht Halt. Trios, die die Besetzung Laute, Violine und Bc aufwiesen, wurden für zwei Lauten und Bc arrangiert, wie z.B. ein Trio von Falckenhagen („die Secund auf die lauten übersetzt“). ${ }^{14}$ Ein Lautenkonzert von Setzkorn wurde so eingerichtet, dass die zweite Laute die erste Violine spielte („so wohl die Principale Lauten als die Primlauten habe abschreiben lassen wie auch die .4. geigen Stim als. Prim Second Alto et Bass“"). ${ }^{15}$ Teilweise waren multiple Besetzungsmöglichkeiten intendiert: So waren die zwei Trios von Andrea Bernasconi mit zwei Lauten alleine, mit zwei Violinen alleine sowie mit Lauten und verstärkenden Violinen spielbar („Prim und Secund auf die Lauten übersetzt wie auch .2. Violin, und Bass extra Copieren lassen“). ${ }^{16}$ Auch Werke für Tasteninstrumente wurden entsprechend arrangiert, so „Von dem Clavicin Vierzehn Trio“, wobei die Violinstimmen für Laute umgeschrieben wurden ("die Prim. auf die Lauten Transponieren lassen“, „dan die Second Violin auf die Lauten auch Transponieren lassen"). ${ }^{17}$ Bei zwei Klavierkonzerten wurden die Solostimme

\footnotetext{
Art. Ferrandini, Giovanni Battista. In Die Musik in Geschichte und Gegenwart. Allgemeine Enzyklopädie der Musik. Ludwig Finscher (Hrsg.). Kassel u.a.: Bärenreiter/Metzler, 2001, Personenteil 6, Sp. 1024-1026. MÜNSTER, Robert. Art. Ferrandini, Giovanni Battista. In Grove Music Online. RISM, <http://www.rism.info/en/service/ opac-search.html>.

10 SADGORSKI, op. cit., S. 55.

11 D-Mbs, Mus.ms.2704: „Sinfonie XXIIII / per / la Capella Elett: / [...] / Del Sig: Wenceslao Wodiczka / Maestro de Concerti / nell'anno 1758.“

$12 \mathrm{Zu}$ einem Lautenisten Setzkorn, der zwischen 1744 und 1750 in Leipzig war vgl. FARSTAD (2000), S. 351.

13 D-Mhsa, KBH 1764, Dok. 114 (1. 2. 1757).

14 D-Mhsa, KBH 1733 II, Dok. 146 (25. 7. 1755).

15 D-Mhsa, KBH 1764, Dok. 114 (1. 2. 1757).

16 D-Mhsa, KBH 1733 II, Dok. 146 (25. 7. 1755).

17 D-Mhsa, KBH 1733 II, Dok. 133 (23. 12. 1754).
} 
und die erste Violine für Lauten eingerichtet („die Concert stim und Prim auf die Lauten übersetzt“) ${ }^{18}$ Ein Klavierstück („ein Clavicin solo“) von Johann Joachim Agrell wurde durch die Ergänzung neukomponierter Violinstimmen zu einem Lautentrio umgearbeitet, das entweder mit Laute und zwei Violinen oder mit drei Lauten oder in gemischter Besetzung spielbar war („darzu habe noch .2. Violin Componieren lassen, solche .3. Sti $[\mathrm{m}] \mathrm{men}$ auf der Lauten Transponiert nehmlich mit .3. Lauten"). ${ }^{19}$ Nach der Heirat Maria Josephas mit dem Markgrafen von Baden-Baden 1755 wurde für Josepha Maria häufig die zweite Violine für Laute umgeschrieben, so dass vor allem Trios von Lautenkomponisten für Laute, Violine und Bass (Paul Charl Durant, Adam Falckenhagen, Karl Kohaut, Johann Kropfgans) mit zwei Lauten besetzt werden konnten.

Schauen wir uns die Lautenkompositionen an, die von italienischen Komponisten stammen, so taucht neben Albinoni Giuseppe Tartini auf, von dem 15 Trios kopiert wurden. Eine Reihe weiterer italienischer Komponisten ließ sich nicht nachweisen und bleibt mehr oder weniger obskur: Berlini (acht Trios), Pianini (sieben Trios, ein Konzert), Vianini (vier Trios) und Vannini (neun Trios), wobei die letzteren drei aufgrund der Ähnlichkeit der Namen auch durchaus identisch sein könnten. Möglicherweise handelt es sich um Ignatius Vianini, ein Kammerdiener von Karl Albrechts Witwe Maria Amalia, der 1751 in ihren Hofstaat kam. ${ }^{20}$ Der ursprünglich aus Padua oder Venedig stammende Würzburger Hofoboist Giovanni Benedetto Platti ist mit drei Trios vertreten.

Auf welchen Transferwegen nun gelangten die italienischen Kompositionen nach München? Dazu lassen sich nur rudimentäre Feststellungen treffen. Während davon ausgegangen werden kann, dass die Trios von Platti, für die nicht festgestellt werden kann, ob es sich um Transkriptionen seiner Trios für Violine, Violoncello und Basso continuo oder andere gehandelt hat, ${ }^{21}$ mittelbar oder unmittelbar aus Würzburg kamen, kann nichts zur Übermittlung der mehr oder weniger obskuren Komponisten Berlini, Pianini und Vannini gesagt werden. Ebenso ist nicht bekannt, welche der 30 publizierten und rund 40 handschriftlich erhaltenen Triosonaten Tartinis ${ }^{22}$ als Vorlage für die Bearbeitung für zwei Lauten gedient haben. In München selbst sind nur wenige Kompositionen Tartinis überliefert, doch sagt dies wenig angesichts der Verluste aus, die die kurfürstliche Musikbibliothek im Laufe der Jahrhunderte erlitten hat. Ein Transfer von Kompositionen ist über Münchner Musiker möglich, die bei Tartini studierten, wie Johann Georg Holzbogen oder Johann Nepomuk Ignaz Lops, die in der Kapelle von Herzog Clemens

18 D-Mhsa, KBH 1733 II, Dok. 146 (25.7.1755).

19 D-Mhsa, KBH 1800, Dok. 121 (12.2.1759).

20 Frdl. Mitteilung von Frank Legl. Es handelt es sich sicherlich nicht um Bernardino oder Elia Vannini (Vanini), die zeitlich nicht in Frage kommen.

2120 Trios in D-WD. Weitere Trio-Kompositionen liegen im Trio für Flöte, Violine und Bc (D-Rtt) und im "Trio con Oboe e Fagotto obligato" und Bc (D-WD) vor.

22 Vgl. BRAINARD, Paul. Le sonate a tre di Giuseppe Tartini. Un sunto bibliografico. Rivista italiana di musicologia, 1969, 4, S. 102-126. 
Franz von Paula von Bayern, einem Cousin des Kurfürsten wirkten, ${ }^{23}$ aber auch über den venezianischen Residenten oder andere Diplomaten des Münchner Hofs. ${ }^{24}$

Albinoni ist hingegen ein sehr interessanter Fall: Hier ist zumindest die Vorlage bekannt: sechs Konzerte in der Besetzung zwei Violinen, Viola und Bc. Ein Blick in das Werkverzeichnis Albinonis zeigt jedoch, dass eine Publikation in der Besetzung eines Concerto a quattro ohne Soloinstrument(e) nicht existiert. Handschriftlich sind hingegen zwölf Balletti und eine Reihe von Sinfonie in vierstimmiger Besetzung bekannt. ${ }^{25}$ Will man nicht davon ausgehen, dass der Eintrag unpräzise ist und Balletti oder Sinfonie gemeint waren oder - wie andere Einträge anscheinend manchmal auch - stillschweigend die Existenz einer Sololaute voraussetzt, so dass man an die Transkription von einigen der zwölf Konzerte op. 9, die Albinoni 1722 Max Emanuel gewidmet hatte und mit ein bis zwei Solooboen, bzw. Solovioline besetzt sind, denken könnte, so ergibt sich noch eine weitere Option. Da das Genre des Concerto a quattro für Streicher ohne Soloinstrument(e) anscheinend in München gepflegt wurde - Evaristo Felice dall'Abaco, Max Emanuels Konzertmeister, widmete dem Kurfürsten 1712 seine Concerti a quattro da chiesa, op. 2 - könnte man vermuten, dass Kompositionen transkribiert wurden, die Albinoni 1722 bei seinem Aufenthalt in München eigens für den kurfürstlichen Hof komponiert hatte. Damals war er nämlich anlässlich der Aufführung seiner Oper I veri amici und seiner Serenata Il trionfo d'amore zur Vermählung Karl Albrechts mit Maria Amalia von Österreich vor Ort ${ }^{26}$ und hätte einen entsprechenden Kompositionsauftrag erledigen können.

Tatsache ist hingegen, dass mit Albinoni Musik arrangiert wurde, die 1754 kaum mehr up-to-date war. Albinoni war nach zweijähriger Bettlägerigkeit 1751 gestorben, seine letzte Oper wurde 1741 in Venedig aufgeführt und seine letzte Publikation erschien 1735/36, ca. 20 Jahre vor den Abrechnungen (op. 10). ${ }^{27}$ Doch wie Johann Joachim Setzkorn berichtete, wurden auch früher schon Werke Albinonis für Laute umgeschrieben - nämlich „etliche Opera“, womit wahrscheinlich die Hochzeitsoper I veri amici und die Serenata Il trionfo d'amore von 1722 gemeint sind -, die zwar älter waren, aber einen Bezug zu München aufwiesen.

23 MÜNSTER (2008), op. cit., S. 71, 76-77. In der Bibliothek des Herzogs waren die ersten beiden Bücher von Tartinis Concerti op. 1 als Druck vorhanden. Ebd., S. 47.

24 Zur Rolle von Diplomaten beim Musiktransfer im 18. Jahrhundert vgl. etwa TRAVERSIER, Mélanie. Costruire la fama musicale. La diplomazia napoletana al servizio della musica durante il regno di Carlo di Borbone. In Europäische Musiker in Venedig, Rom und Neapel (1650-1750) / Les Musiciens européens à Venise, Rome et Naples (1650-1750) / Musicisti europei a Venezia, Roma e Napoli (1650-1750). Anne-Madeleine Goulet - Gesa zur Nieden (Hrsg.). Kassel: Bärenreiter, 2015 (= Analecta musicologica 52), S. 171-189.

25 Balletti u.a. in A-Wn, EM.94/a, E.M.94/b, US-BEm, Italian MS 63; Sinfonie u.a. in A-Wn, D-Dl, S-L, SSkma, S-Uu, D-DS (zweifelhaft) nach TALBOT, Michael. Art. Albinoni, Tomaso. In Grove Music Online (konsultiert 18. Oktober 2017). RISM Online Catalogue of Musical Sources. URL: <www.rism.info〉. ROSSI, Franco. Catalogo tematico delle composizioni di Tomaso Albinoni (1671-1750). Parte II: Le opere strumentali manoscritte. Le opere vocali. I libretti. Padua: Edizione di „I solisti veneti“, 2003, S. 244-308.

26 TALBOT, Michael. Albinoni. Leben und Werk. Adliswil: Kunzelmann, 1980, S. 52-54.

27 Zu den späten Jahren Albinonis vgl. TALBOT (1980), op. cit., S. 60-70. Eine weitere Publikation (op. 11) war 1739 in Planung, zu einer Veröffentlichung kam es jedoch nicht. TALBOT (Art. Albinoni), op. cit. 
Ohnehin ist die Überlieferung der Bearbeitungen ein Problem. Denn von dem um die 500 Kompositionen umfassenden Bestand ist nichts überliefert. Lediglich eine Handschrift, die in den Abrechnungen nicht auftaucht, könnte etwas mit dem in München gepflegten Lautenrepertoire zu tun haben. Sie liegt in der Musikabteilung der Bayerischen Staatsbibliothek:

D-Mbs, Mus.ms.504: „LIBRO / DELLA / MANDORA / E / Fundamento della Mand:“ ist eine Sammlung von sechs Partiten, einem Divertimento und einem Menuett für Mandora eines anonymen Komponisten. Diese Sammlung trägt das monochrome Wappen Kaiser Franz Stephans von Lothringen (des Ehemanns von Kaiserin Maria Theresia) auf der Titelseite sowie das ebenfalls monochrome bayerische Wappen auf der Rückseite und könnte daher etwas mit der 1765 erfolgten Vermählung Josepha Marias mit dem kaiserlichen Thronfolger Joseph zu tun haben. ${ }^{28}$ Franz Stephan starb kurz darauf, so dass ihm sein Sohn als Joseph I. im gleichen Jahr nachfolgte, bzw. er als Mitregent seiner Mutter den Thron bestieg. Die Mandora-Schule richtete sich insbesondere an Frauen, wie aus dem fragmentarischen Vorwort hervorgeht: „Gleichwie sich aber bishero noch niemand bemühet hat, den Tact, und die Griffe, welche die Jenigen, So der Music unerfahren sind, insonderheit die Frauen= [es fehlen eine oder mehrere Seiten].“

Weitere Kompositionen für Zupfinstrumente stammen ebenfalls offensichtlich aus München und sind in Dresden aus dem Besitz Kurfürstin Maria Antonia Walpurgis' überliefert, die als Wittelsbacherin 1747 Friedrich Christian von Sachsen geehelicht hatte. Im Katalog ihrer Musiksammlung ist „1 Paquetto Musica per il Gallichon“ verzeichnet; ${ }^{29}$ das Calichon, das auch als Mandora bezeichnet wurde, ist ein Zupfinstrument, das gerade im 18. Jahrhundert sehr beliebt war. ${ }^{30}$

D-Dl, Mus.2701-V-1: „Tre Serenate / Per il Gallichona / De S: A. Duce Clemente / Di Baviera" enthält Werke für Calichon und neben den drei Serenaden von Herzog Clemens Franz von Paula eine „Polacca“ sowie zwölf Menuette des Kurfürsten Max III. Joseph.

D-D1, Mus.3037-D-1, Mus.3037-D-1a, Mus.3037-V-3: die „Cantata sacra“ O spettacolo pur troppo funesto, eine Passionskantate des Münchner Kammermusikdirektors Giovanni Battista Ferrandini, ist mit Sopran, zwei Gamben, Laute und Bc besetzt und muss zwischen 1742 und 1745 entstanden sein, denn auf dem Innentitel der Vokalpartitur für Sopran und Bc findet sich folgender Hinweis: „Cantata Sacra / di Giovanni Ferrandini / Consigliere / e Maestro di Capella / di S. M. Carlo VII / Imperadore." Ferrandini wurde 1737 zum Rat und Kammermusikdirektor ernannt; Karl Albrecht von Bayern, seit 1742 Kaiser Karl VII., starb 1745. Wäre die Komposition und die Handschrift unter seinem Nachfolger entstanden, wäre der Titel sicherlich anders ausgefallen. Möglicherweise ist die Kantate mit dem Posten „Cantata Sancta auss die Charwoch[en] Verstörckht" in Verbindung zu bringen, der 1758 für Josepha Maria in Rechnung gestellt wurde und zeigt, dass ein

28 Auf der Titelseite findet sich in einer Banderole die Jahreszahl (?) 15 38, die Handschrift stammt aber unzweifelhaft aus dem 18. Jahrhundert.

29 Vgl. RISM ID no.: 212006918.

30 Vgl. die Artikel KIRSCH, Dieter. Calichon und TYLER, James. Mandora in Grove Music Online. SAYCE, Lynda. A Forgotten Lute: a Painless Introduction to 18th Century Lute Music. Lute News, 2003, 67, S. 7-10. 
vorhandenes Manuskript buchbinderisch gerichtet wurde. ${ }^{31}$ Es könnte sich jedoch auch um eine andere Komposition gehandelt haben. O spettacolo pur troppo funesto spiegelt die „Musikkompetenz“ der Münchner Regenten wider: Max III. Joseph spielte Gambe, sein Vater Karl Albrecht Laute, seine Schwester Maria Antonia Walpurgis sang, komponierte bekanntlich und spielte ebenfalls Laute. ${ }^{32}$ Denn ihre Mutter Maria Amalia tauschte sich 1747 mit ihr darüber aus:

„Ich hab gehörtd, dass du dich auch zuweilen noch auf daß lautenschlagen begibst, welches wohl ohne zweifl bë̈ dem berühmten [Silvius Leopold] weiss sein wirdt möchte gern einige Partien oder stuck von seiner Composition vor die Marie [Josepha] haben, dan dise vill besser auf dem Gusto wie es sich auf dises instrument gehördt, Componirt sein, als alle Krazereÿen von Sezkorn." ${ }^{33}$

Diese Briefstelle zeigt zudem, dass Maria Josepha bereits einige Jahre vor dem Beginn der Abrechnungen 1751 in Laute unterrichtet wurde und auch damals schon Johann Joachim oder Ferdinand Maria Setzkorn eine wichtige Rolle spielten.

In den oben angesprochenen Kontext fürstlicher Musikpraxis ist sicherlich auch das „Musicale intratenimento“ oder „Musicale tratenimento“ für Violine, zwei Lauten, Viola da gamba und Bc von Ferrandini einzuordnen, das laut $M G G$ und New Grove in D-Dl überliefert sein soll, sich über RISM und den Online-Katalog der Sächsischen Landes- und Universitätsbibliothek allerdings nicht nachweisen ließ.

Dresdner Handschriften zeigen außerdem, dass sich die Bearbeitungspraktiken am sächsischen Hof nicht von denen in München unterschieden. Denn die „Sonate / a due Gallichane. [!]“ von Johann Paul Schiffelholz (D-Dl, Mus.2806-V-6) basieren auf einer Version für Violine, Calichon und Bc (D-Dl, Mus.2806-V-2a (II) bis Mus.2806-V-2a (VI) und Mus.2806-V-2). Diese Werke stammen ebenfalls vermutlich aus Maria Antonia Walpurgis' Besitz.

Während also keine Bestände der dargestellten Musikpraxis in der Münchner fürstlichen Kammer überliefert sind, bieten uns die Dresdner Quellen zumindest eine Ahnung davon. Über die Transfers von Musik nach München sagen diese Quellen indessen nichts aus. Doch mit Maria Antonia Walpurgis haben wir vermutlich eines der Scharniere in der Übermittlung „sächsischen Repertoires“, wie es uns in den Werken von Johann Georg Neruda und Johann Kropfgans, die in Dresden und Leipzig wirkten, begegnet. ${ }^{34}$

31 D-Mhsa, KBH 1781, Dok. 114.

32 Zusammenfassend FISCHER, Christine. Instrumentierte Visionen weiblicher Macht. Maria Antonia Walpurgis' Werke als Bühne politischer Selbstinszenierung (= Schweizer Beiträge zur Musikforschung 7). Bärenreiter: Kassel u.a., 2007, S. 7-18. ALLROGGEN, Gerhard. Art. Maria Antonia Walpurgis, Electress of Saxony. In Grove Music Online. MÜNSTER, Robert. Art. Maria Antonia Walpurgis Symphorosa. In Die Musik in Geschichte und Gegenwart. Allgemeine Enzyklopädie der Musik. Ludwig Finscher (Hrsg.). Kassel u.a.: Bärenreiter/Metzler, 2004, Personenteil 11, Sp. 1085-1086.

33 Das Dokument befindet sich in D-Dla, Hausarchiv, Marie Antonie, Nr. 2a: Briefe an die Kurprinzessin [nachmalige Kurfürstin] Marie Antonie von ihrer Mutter, Nr. 1-89, Brief Nr. 23 vom 5. Oktober 1747, Bl. 52b (frdl. Mitteilung von Frank Legl); erstmals veröffentlicht in: NEEMANN, Hans. Die Lautenistenfamilie Weiß. Archiv für Musikforschung, 1939, 4, S. 157-189: 169 (ohne Quellenangabe).

34 SCHERING, Arnold. Musikgeschichte Leipzigs. Bd. 2: Von 1650 bis 1723 (= Schriften der Königlich-Sächsischen Kommission für Geschichte 30), Leipzig: Teubner, 1926, S. 413 f. Bd. 3: Von 1723 bis 1800 (= Schriften der Königlich-Sächsischen Kommission für Geschichte 40). Leipzig: Teubner, 1941, S. 548 f. FARSTAD (2000), 
Musik aus Bayreuth von Adam Falckenhagen und Charl Durant könnte ebenfalls direkt bezogen worden sein, denn in den 50er Jahren scheinen intensivere musikalische Beziehungen nach Bayreuth bestanden zu haben, da Andrea Bernasconis 1754 dort aus Anlass des Besuchs Friedrichs des Großen aufgeführte festa teatrale L'huomo wohl ein Auftragswerk war. ${ }^{35}$ Durant war überdies 1748 von Regensburg nach München gereist ${ }^{36}$ und könnte innerhalb seines professionellen Netzwerks entsprechende Kontakte geknüpft haben. Tartinis Kompositionen könnten auf direktem Wege von Münchner Musikern, die bei Tartini Unterricht nahmen, in die bayerische Residenzstadt gelangt sein. Auch die Rolle von Mittelsmännern, Netzwerken und Diplomaten darf nicht unterschätzt werden. ${ }^{37}$ Andere Kompositionen könnten über Drucke in das Repertoire gelangt sein wie Trios von Philipp Martin, die zwischen 1730 und 1733 in Augsburg publiziert worden waren. ${ }^{38}$ Wie Wiener Musik von Karl Kohaut ${ }^{39}$ oder Wagenseil nach München gelangte, ist nicht zu klären. Die Habsburgerin Kurfürstin Maria Amalia scheidet als Vermittlerin aus, da sie bereits 1756 starb, Kohauts und Wagenseils Musik jedoch erst ab 1761 in das Lautenrepertoire gelangten. Für Albinonis Konzerte schließlich ist die Überlegung legitim, dass sie genuin für München entstanden sein könnten. Insgesamt ist aber neben einem geographischen Transfer ein musikalischer durch das Arrangement auf die spezifischen Bedürfnisse eines „Nischenrepertoires“ zu konstatieren - eines Repertoires, das für musikalische Laien spielbar war, wenn auch ihr Status als bayerische Prinzessinnen sie allen egalisierenden Sphären enthob.

\section{Bibliography}

\section{Sources}

D-Dl, Mus.2701-V-1: „Tre Serenate / Per il Gallichona / De S: A. Duce Clemente / Di Baviera.“

D-Dl, Mus.2806-V-2a (II) bis Mus.2806-V-2a (VI), Mus.2806-V-2: Johann Paul Schiffelholz, Trios

D-Dl, Mus.2806-V-6: Johann Paul Schiffelholz, „Sonate / a due Gallichane. [!]“

D-Dl, Mus.3037-D-1, Mus.3037-D-1a, Mus.3037-V-3: „Cantata Sacra / di Giovanni Ferrandini / Consigliere / e Maestro di Capella / di S. M. Carlo VII / Imperadore."

D-Dla, Hausarchiv, Marie Antonie, Nr. 2a: Briefe an die Kurprinzessin [nachmalige Kurfürstin] Marie Antonie von ihrer Mutter, Nr. 1-89.

D-Mbs, Mus.ms.504: „LIBRO / DELLA / MANDORA / E / Fundamento della Mand:“

op. cit., S. 333 f., 340 .

35 SADGORSKI, op. cit., S. 58.

36 KIRÁLY, Peter. Quellenangaben zu Paul Charl(es) Durants möglicher Abstammung. Die Laute. Jahrbuch der Deutschen Lautengesellschaft, 2003, 7, S. 78-82. LEGL, Frank. Neue Quellen zur Lautenistenfamilie Weiss, Paul Charl Durant und Wolff Jacob Lauffensteiner. Die Laute. Jahrbuch der Deutschen Lautengesellschaft, 2011, 9-10, S. 11-40: 18-20. FARSTAD (2000), op. cit., S. 301-303, 319.

37 Vgl. dazu etwa die in LEGL, op. cit., erwähnten Namen.

38 Vgl. FARSTAD (2000), op. cit., S. 338

39 Vgl. FARSTAD (2000), op. cit., S. 331 f. 
D-Mbs, Mus.ms.2704: „Sinfonie XXIIII / per / la Capella Elett: / [...] / Del Sig: Wenceslao Wodiczka / Maestro de Concerti / nell'anno 1758."

D-Mhsa, Kurbayern HR I, Fasz. 41.

D-Mhsa, Kurbayern Hofzahlamt (KBH) 1663 (1751), 1699 (1753), 1718 (1754), 1733 II (1755), 1764 (1757), 1781 (1758), 1800 (1759), 1818 (1760), 1833 (1761), 1850 (1762), 1870 (1763), 1883 (1764).

\section{Literature}

ALLROGGEN, Gerhard. Art. Maria Antonia Walpurgis, Electress of Saxony. In Grove Music Online. BÖHMER, Karl. Art. Ferrandini, Giovanni Battista. In Die Musik in Geschichte und Gegenwart. Allgemeine Enzyklopädie der Musik. Ludwig Finscher (Hrsg.). Kassel u.a.: Bärenreiter/Metzler, 2001, Personenteil 6, Sp. 1024-1026.

BRAINARD, Paul. Le sonate a tre di Giuseppe Tartini. Un sunto bibliografico. Rivista italiana di musicologia, 1969, 4, S. 102-126.

FARSTAD, Per Kjetil. German Galant Lute Music in the 18th Century. A Study of the Period, the Style, Central Lutenists, Ornaments, Idiomatic, and Problems that Arise When Adapting Lute Music from this Period to the Modern Eight-Stringed Classical Guitar. Göteborg: Göteborg Univ., Dep. of Musicology, 2000.

FARSTAD, Per Kjetil. German Galant Lute Music. Guitar Review, 2001, 123, S. 10-23.

FISCHER, Christine. Instrumentierte Visionen weiblicher Macht. Maria Antonia Walpurgis' Werke als Bühne politischer Selbstinszenierung (= Schweizer Beiträge zur Musikforschung 7). Bärenreiter: Kassel u.a., 2007.

KIRÁLY, Peter. Quellenangaben zu Paul Charl(es) Durants möglicher Abstammung. Die Laute. Jahrbuch der Deutschen Lautengesellschaft, 2003, 7, S. 78-82.

KIRSCH, Dieter. Calichon. In Grove Music Online.

LEGL, Frank. „der alte Weiß spielte“ - Laute und Theorbe in der zweiten Hälfte des 18. und zu Beginn des 19. Jahrhunderts. In Laute und Theorbe. Symposium im Rahmen der 31. Tage Alter Musik in Herne 2006. Stadt Herne (Hrsg.), Konzeption und Redaktion Christian Ahrens - Gregor Klinke. München und Salzburg: Katzbichler, 2009 S. 30-49.

LEGL, Frank. Johann Joachim Setzkorn und ein Corpus verschollener Musik für und mit Laute. In Die Laute. Jahrbuch der Deutschen Lautengesellschaft (i.Vorb.).

LEGL, Frank. Neue Quellen zur Lautenistenfamilie Weiss, Paul Charl Durant und Wolff Jacob Lauffensteiner. Die Laute. Jahrbuch der Deutschen Lautengesellschaft, 2011, 9-10, S. 11-40.

MÜNSTER, Robert. Die Musk am Hofe Max Emanuels. In Kurfürst Max Emanuel. Bayern und Europa um 1700. Bd. 1: Zur Geschichte und Kunstgeschichte der Max-Emanuel-Zeit. Hubert Glaser (Hrsg.). München: Hirmer, 1976, S. 295-316.

MÜNSTER, Robert. Art. Ferrandini, Giovanni Battista. In Grove Music Online.

MÜNSTER, Robert. Herzog Clemens Franz von Paula von Bayern (1722-1790) und seine Münchner Hofmusik. Tutzing: Schneider, 2008.

MÜNSTER, Robert. Art. Maria Antonia Walpurgis Symphorosa. In Die Musik in Geschichte und Gegenwart. Allgemeine Enzyklopädie der Musik. Ludwig Finscher (Hrsg.). Kassel u.a.: Bärenreiter/ Metzler, 2004, Personenteil 11, Sp. 1085-1086.

NEEMANN, Hans. Die Lautenistenfamilie Weiß. Archiv für Musikforschung, 1939, 4, S. 157-189.

OVER, Berthold. ,...sotto l'Ombra della Regina di Pennati‘. Antonio Vivaldi, Kurfürstin Therese Kunigunde von Bayern und andere Wittelsbacher. In Italian Opera in Central Europe 1614-1780. 
Bd. 3: Opera Subjects and European Relationships. Norbert Dubowy - Corinna Herr - Alina Zorawska-Witkowska (Hrsg.). Berlin: BWV - Berliner Wissenschafts-Verlag, 2007, S. 251-297.

PÄFFGEN, Peter. Ein Artliches Lob der Lauten. Blüte und Niedergang von Laute und Lautenspiel im 16.-18. Jahrhundert. Concerto. Das Magazin für Alte Musik, 1985, 2, S. 48-55.

PERUTKOVÁ, Jana. Der glorreiche Nahmen Adami. Johann Adam Graf von Questenberg (1678-1752) als Förderer der italienischen Oper in Mähren. Wien: Hollitzer, 2015.

RISM Online Catalogue of Musical Sources. URL: <www.rism.info>.

ROSSI, Franco. Catalogo tematico delle composizioni di Tomaso Albinoni (1671-1750). Parte II: Le opere strumentali manoscritte. Le opere vocali. I libretti. Padua: Edizione di „I solisti veneti“, 2003.

SADGORSKI, Daniela. Andrea Bernasconi und die Oper am Münchner Kurfürstenhof 1735-1772. München: Herbert Utz Verlag, 2010.

SAYCE, Lynda. A Forgotten Lute: a Painless Introduction to 18th Century Lute Music. Lute News, 2003, 67, S. 7-10.

SCHERING, Arnold. Musikgeschichte Leipzigs. Bd. 2: Von 1650 bis 1723 (= Schriften der KöniglichSächsischen Kommission für Geschichte 30), Leipzig: Teubner, 1926.

SCHERING, Arnold. Musikgeschichte Leipzigs. Bd. 3: Von 1723 bis 1800 (= Schriften der KöniglichSächsischen Kommission für Geschichte 40). Leipzig: Teubner, 1941.

TALBOT, Michael. Albinoni. Leben und Werk. Adliswil: Kunzelmann, 1980.

TALBOT, Michael. Art. Albinoni, Tomaso. In Grove Music Online.

TRAVERSIER, Mélanie. Costruire la fama musicale. La diplomazia napoletana al servizio della musica durante il regno di Carlo di Borbone. In Europäische Musiker in Venedig, Rom und Neapel (1650-1750) / Les Musiciens européens à Venise, Rome et Naples (1650-1750) / Musicisti europei a Venezia, Roma e Napoli (1650-1750). Anne-Madeleine Goulet - Gesa zur Nieden (Hrsg.). Kassel: Bärenreiter, 2015 (= Analecta musicologica 52), S. 171-189.

TYLER, James. Mandora. In Grove Music Online.

WERR, Sebastian. Politik mit sinnlichen Mitteln. Oper und Fest am Münchner Hof (1680-1745). Köln u.a.: Böhlau, 2010.

ZEDLER, Andrea. Alle Glückseligkeit seiner Education dem allermildesten Ertz-Hause Oesterreich zu dancken“. Hofstaat, Bildung und musikalische Unterweisung des bayerischen Kuprinzen Karl Albrecht in Graz (1712-1715). Historisches Jahrbuch der Stadt Graz, 2012, 42, S. 337-366. 